\title{
Radiotherapy or axillary lymph node dissection in breast cancer patients with positive sentinel lymph nodes?
}

\author{
Aleksandra Łacko ${ }^{1,2}$, Wojciech M. Wysocki ${ }^{3}$
}

Over the years the surgical management of localized breast cancer has become less aggressive. Complete resection of the primary tumour with negative margins replaced mastectomy and is currently a standard of care in a vast majority of women with early breast cancer. Also, an introduction of sentinel lymph node biopsy allowed for giving up axillary lymph node dissection in the patients with pathologically negative sentinel nodes. This development has led to a decrease of morbidity such as lymphedema. However, the standard management of axilla in the patients with positive sentinel lymph nodes remains controversial. Recent studies demonstrated that in the patients with 1 or 2 positive sentinel lymph nodes who undergo breast conserving surgery followed by conventionally fractionated whole-breast radiotherapy, a completion axillary lymph node dissection can be avoided. Furthermore, evidence from three two-phase non-inferiority studies comparing radiotherapy with complete lymph node dissection did not show any significant differences in either overall and disease-free survival, or local control and decrease of the percentage of patients with local adverse effects in radiotherapy arms. However, there are several methodological drawbacks and clinical limitations of these studies, which prevent from general omission of completion axillary lymph node dissection in breast cancer patients with positive sentinel lymph nodes. The debate held during V Annual Conference of Nowotwory Journal of Oncology and resulting article discusses the optimal management of axilla in this population.

NOWOTWORY J Oncol 2018; 68, 1: 38-41

Key words: breast cancer, axillary lymph node dissection, radiotherapy, sentinel lymph node

\section{Introduction}

Lower aggressiveness of surgical treatment of early breast cancer is connected with a smaller mutilation of a patient, yet the treatment efficiency is, at the same time, preserved. In the last three decades, the tendency for the minimalization of the scope of surgical treatment has concerned also the interventions in the axillary fossa. The change of the standard of care is so significant that in literature it is frequently referred to as "revolution". Nevertheless, such term seems to be slightly premature, as still there is a number of doubts concerning the indications for "conserving" treatment of the axillary fossa.

The first milestone in the minimalization of the axillary fossa treatment was the general application of sentinel lymph node biopsy (SNB) in the patients without clinically enlarged lymph nodes (CNO). In the case of a lack of metastases, such a procedure allows for a resignation from an axillary lymph node dissection (ALND), which saves the patients from complications connected with such an intervention, lymphedema in particular [1]. Another significant change in the surgical standard of care was the resignation from the surgical treatment of the axillary fossa in the patients with 1 or 2 metastases in sentinel nodes, treated with conserving treatment. Two clinical trials, ACOSOG Z0011 and IBCSG 23-01 showed that the abandonment of a surgical treatment in this group of patients, in comparison with ALND guaranteed a comparable local control and did not affect the survival period [2,3]. It must be stressed that both trials

\footnotetext{
${ }^{1}$ Department of Oncology, Medical University, Wrocław, Poland

${ }^{2}$ Lower Silesia Oncology Centre, Wrocław, Poland

${ }^{3}$ Department of Surgical Oncology, Maria Skłodowska-Curie Memorial Cancer Centre and Institute of Oncology, Kraków Branch, Poland
} 
concerned the patients with favourable prognostic features: in about $80 \%$ patients in the ACOSOG Z0011 trial and in 95\% patients in the IBCSG 23-01 trial, only one metastatic sentinel node was found, whilst only micro-metastases were present in $40 \%$ and $100 \%$, in ACOSOG Z0011 and IBCSG 2301 respectively. Thus, there is no confidence that this is also the optimum procedure in a group with poorer prognoses. Moreover, since $100 \%$ in the ACOSOG Z0011 study and $90 \%$ women in the IBCSG 23-01 study, were treated with conserving treatment with tangential field radiotherapy (RT), the conclusions from these studies are not definite and it cannot be ruled out that it was irradiation that compensated for an inadequate scope of the surgery. Therefore, indiscriminate resignation from ALND after a positive sentinel node biopsy in every patient does not seem rational or justified.

It must be observed that SNB is performed also in the patients with locally advanced breast cancer without clinically involved sentinel nodes on the axilla, after systemic pre-operative treatment, mastectomy patients in whose case no adjuvant RT is planned and also in a situation of a local recurrence after a previous conserving treatment. All the patients with such characteristics were not included in the above trials and that is why, in the case of a confirmation of SLN metastases, there are no grounds to abandon ALND, or to replace a surgical intervention with RT [4]. The exception here concerns the patients after mastectomy with micro-metastases in sentinel lymph nodes. This recommendation is based on the results of the two studies concerning women after mastectomy, which compared adjuvant ALND with the abandonment of further treatment $[5,6]$. Neither of the studies showed any differences with regards to progression free survival.

In the patients with an increased risk of recurrence (tumour $\geq 3 \mathrm{~cm}$, lymphatic involvement or micro-infiltration of the lymph node capsule) the optimal treatment is also controversial. In such a situation, the resignation from ALND seems to be possible, yet RT must be applied in the axillary fossa [7].

With regards to the indisputably lower risk of complications connected with radiotherapy, in comparison with long-term consequences of axillary lymphadenectomy, the next objective of the clinical studies was the comparison of both local treatment methods in the patients with early breast cancer.

\section{Randomized studies comparing radiotherapy versus ALND in women with positive sentinel lymph node}

The concept of the resignation from the surgical treatment of the axillary fossa and the replacement of the surgical intervention with irradiation was by no means new, as in the past it was evaluated in two separate clinical studies carried out in the 1970s and 1980s. In both cases no diffe- rences with respect to the overall survival and progression free survival were shown in the patients, although in the study carried out by Louis-Sylvestre et al., the frequency of local recurrences was significantly larger in the patients undergoing radiotherapy ( $3 \%$ vs $1 \% ; p=0.04$ ) [8]. A great advantage of both studies is a long follow-up period, whilst the disadvantage, rendering the interpretation of the results more difficult, lies in the significant differences in the characteristics of the patients (larger advancement stage) and in the administered treatment (both surgical and systemic ones - in comparison with the contemporary methods). The main objective of the NSABP B-04 study was the comparison of Halsted's mastectomy with mastectomy alone or mastectomy completed with irradiation [9]. No patient in the study was treated systematically. In the light of the current standards, such procedure would be regarded as suboptimal. Therefore, the results of two "old" studies quoted in this paragraph should not provide the grounds for the clinical decisions taken in contemporary settings (certainly only in the treatment area discussed herein).

In two other "contemporary" studies - a multi-centre, international and randomised phase III study, AMAROS and a single-centre Hungarian, phase III study, OTOASOR adjuvant RT was compared with ALND in the patients with metastases in the sentinel lymph nodes $[10,11]$. Both clinical trials are the studies of the non-inferiority type. The results of the analyses carried out after 6 and 8-year follow-up period, in the AMAROS and OTOASOR study, respectively, point to comparable treatment results in both groups of patients with regards to survival indicators and local control. What draws attention, however, is a very small number of recurrences in the axillary fossa, irrespectively of the type of treatment (Table I). Some significant differences, however, concerned the long-term complications after the treatment. In the AMAROS study, in the patients undergoing radiotherapy, a limb lymphoedema was observed twice less often ( $40 \%$ vs $21,7 \%$ after one year, $28 \%$ vs $13,6 \%$ after two years, in the arm with ALND and RT respectively). In the evaluation of the quality of life, the differences were observed solely in

Table I. The Z0011 [3] and AMAROS studies [10] and OTOASOR [11]

\begin{tabular}{|c|c|c|c|}
\hline & Z0011 & AMAROS & OTOASOR \\
\hline Median number of resected SN & 2 & $2(1-3)$ & $2(1-5)$ \\
\hline Macro-metastases & $50 \%$ & $60 \%$ & $60 \%$ \\
\hline Additional lymph nodes after ALND & $27.3 \%$ & $32.8 \%$ & $38.5 \%$ \\
\hline Recurrence in axillary fossa: ALND & $0.5 \%$ & $0.43 \%$ & $1.7 \%$ \\
\hline Recurrence in axillary fossa: follow-up & $1.5 \%$ & $1.19 \%$ & $2 \%$ \\
\hline Follow-up median & 9.25 years & 6.1 years & 8 years \\
\hline $\mathrm{BCT}$ & $100 \%$ & $82 \%$ & $84 \%$ \\
\hline
\end{tabular}


the assessment of the symptoms concerning the upper limb. Both better mobility of the limb and less intensive oedemas of the hand and the limb point to the benefit of irradiation. In the OTOASOR study, the differences in the frequency of the upper limb symptoms (lymphoedema, paraesthesia, pain, mobility impairment) between both groups were even larger (15.3\% vs $4.7 \%$, in ALND and radiotherapy groups respectively).

The patient populations were in both studies alike. The majority were the post-menopausal women with well-differentiated ductal carcinomas and hormone receptors expression. Although the tumour size differed significantly in both protocols (the OTOASOR study concerned the patients with tumours $\leq 3 \mathrm{~cm}$, and the AMAROS study $<\mathrm{T} 2$ ), in reality the majority of patients in both groups were the women with tumours $<2 \mathrm{~cm}$ ( $62 \%$ and $82 \%$, in the OTOASOR and AMAROS study respectively). Also the share of the patients treated with breast conserving treatment as compared with the mastectomy group was almost identical (20\% and $16-18 \%$ in the OTOASOR and AMAROS study respectively). In both studies, systemic treatment was carried out according to the current standards.

The criticism of both studies concerned a short follow-up period, a small number of events, a population with good prognoses and a lack of the analyses of the subgroups with respect to the biological characteristics of the tumour. With regards to a small number of events in the AMAROS study (assumed recurrence number: $2 \%$, real number of recurrences: $0.43 \%$ in the group with ALND and $1.19 \%$ in the group with RT) the equivalence of both treatment methods could not be confirmed, which weakens the strength of the results. Moreover, in the OTOASOR study, the analysis of the efficacy was of the per protocol type, instead of the required non-inferiority intention-to-treat analysis (i.e. in the groups selected in accordance with the planned treatment). The results of the AMAROS study could have also been affected by the scope of the local treatment. In accordance with the protocol, ALND covered only the first and second level of the axillary fossa, whilst adjuvant RT — all the tree levels of the axillary fossa plus supraclavicular area. Therefore the scope of anatomic effect of both local treatment methods compared in the study was different: it was smaller in the case of a surgery and larger in the case of RT.

Axillary radiotherapy in the patients with positive SN is still controversial. Due to the lack of high level evidence, little is known about which patients require RT. Two studies examined this issue. In MA.20 [12] and EORTC 22922-10925 [13] comparing breast irradiation alone versus radiotherapy of the breast and node fields showed significant decrease in locoregional recurrence and distant spread, and $1 \%$ to $2 \%$ overall survival benefit at 10 years. However, due to a relatively modest benefit of radiotherapy patients with low risk disease and limited nodal burden are likely to be over- treated. In prospective study patients with positive sentinel lymph node meeting ACOSOG ZOO11 study characteristics were treated with SNB alone. After 5 years nodal recurrences were observed in only $1.4 \%$ of patients [14].

\section{Axillary management after neoadjuvant therapy}

The increasing use of neoadjuvant therapy, in both high-risk clinically node negative (cNO) disease and in clinically node positive ( $\mathrm{cN} 1$ ) disease, potentially offers an opportunity to avoid ALND, because neoadjuvant therapy may result in downstaging of the axilla. However, the decision regarding the surgical management of the axilla must take into account several factors such as the performance characteristics of the SLN procedure after neoadjuvant therapy, the molecular subtype of the primary tumour and the planned breast procedure. For the patients with aggressive breast cancer subtypes such as triple negative or HER2+ disease, the use of neoadjuvant therapy is associated with high rates of nodal $\mathrm{pCR}$. In patients with $\mathrm{cN} 1$ converted to CNO, SLN procedure is to be performed, and axillary node dissection may be omitted. An intriguing thing is that in cNO patients meeting eligibility criteria for avoidance of ALND in the trials of upfront surgery, the use of neoadjuvant therapy may not significantly impact rates of ALND [15]. However, retrospective analysis performed in 1980 women showed that patients with most aggressive subtypes, triple negative, and HER-2 positive BC, treated with neoadjuvant therapy and mastectomy, the need for ALND was significantly reduced [15]. For patients with advanced cN1, luminal, HER-2 negative disease, neoadjuvant therapy may result in downstaging of the axilla which offers more opportunity for individualized management [16]. Currently, endocrine neoadjuvant therapy is increasingly used in patients with low and intermediate grade, node positive, luminal tumours. The data regarding the optimal axilla management after neoadjuvant endocrine therapy are limited. Thus patient selection for SN after preoperative systemic treatment is critical. As patients treated with neoadjuvant therapy were not included in the studies confirming safety and efficacy of SNB alone, women with positive SN still require ALND.

\section{Conclusions}

In the debate during the last St. Gallen experts' meeting in Vienna in 2017 concerning the optimal standard of care in the patients with metastases present in 1-2 lymph nodes after breast conserving treatment in whose case irradiation is planned with the application of both standard and high tangential field radiotherapy, almost $80 \%$ respondents voted for the resignation from ALND. In the post-mastectomy patients, ALND was regarded as indicated solely in a situation when no adjuvant radiotherapy was planned. The changes in the standard of the local treatment of axillary fossa are currently introduced and new data systematically 
provide arguments for the resignation from an adjuvant surgery of the axillary fossa. This is understandable in a situation when two treatment methods with comparable efficacy are available. By all means, the choice of a method with a lower risk of recurrence (out of the available treatment methods) is justified and indicated. Radiotherapy, and in some cases only observation, replaces lymphadenectomy on account to the significantly lower risk of long-term treatment complications and better quality of life confirmed in a long follow up period. This concerns the majority of patients meeting the inclusion criteria for the AMAROS and OTOASOR studies. Certainly a question remains whether in all the patients the minimalization of the surgical intervention in the axillary fossa is indicated. The results of these studies do not concern all the patients with an involvement of the sentinel nodes (among others - the patients with preoperative systemic treatment, the patients undergoing mastectomy, in whose case no RT is planned or the treated for local recurrences). In such cases, still ALND is recommended. It seems that in the decisions concerning the local treatment of the axillary fossa, it is significant to take into consideration such factors as the biology of the tumour, the type of systemic treatment and the scope of the planned radiotherapy.

\section{Conflict of interest: none declared}

\section{Wojciech M. Wysocki, MD, PhD}

Maria Skłodowska-Curie Memorial Cancer Centre and Institute of Oncology

Kraków Branch

Department of Surgical Oncology

ul. Garncarska 11, 31-115 Kraków, Poland

e-mail:z5wysock@cyf-kr.edu.pl

Received \& Accepted: 20 Apr 2018

Based on the presentation at the V Annual Conference of the Nowotwory Journal of Oncology, 'Oncological Debates, held in Warszawa, 7-8th April 2017

\section{References}

1. Krag DN, Anderson SJ, Julian TB et al. Sentinel-lymph-node resection compared with conventional axillary-lymph-node dissection in clinically node-negative patients with breast cancer: overall survival findings from the NSABP B-32 randomised phase 3 trial. Lancet Oncol 2010; 11: 927-933.

2. Giuliano $\mathrm{AE}$, Hunt $\mathrm{KK}$, Ballman $\mathrm{KV}$ et al. Axillary dissection vs no axillary dissection in women with invasive breast cancer and sentinel node metastasis: a randomized clinical trial. JAMA 2011; 305: 569-575.

3. Giuliano $A E, M c C a l l ~ L$, Beitsch $P$ et al. Locoregional recurrence after sentinel lymph node dissection with or without axillary dissection in patients with sentinel lymph node metastases: the American College of Surgeons Oncology Group Z0011 randomized trial. Ann Surg 2010; 252: 426-433.

4. Lyman GH, Temin S, Edge SB et al. Sentinel lymph node biopsy for patients with early-stage breast cancer: American Society of Clinical Oncology clinical practice guideline update. J Clin Oncol 2014; 32: 1365-1383.

5. Galimberti V, Cole BF, Zurrida $S$ et al. Axillary dissection versus no axillary dissection in patients with sentinel-node micrometastases (IBCSG 23-01): a phase 3 randomised controlled trial. Lancet Oncol 2013; 14: 297-305

6. Sola M, Alberro JA, Fralle M et. al. Comlete axillary lymph node dissection versus clinical follow-up in breast cancer patients with sentine node micrometastasis: final results from the multicenter clinical trial AATRM 048/13/2000. Ann Surg Oncol 2013; 20: 120-127.

7. 7.Morrow M. Management of the node-positive axilla in breast cancer in 2017: Selecting the right option. JAMA Oncol 2018; 4: 250-251.

8. Louis-Sylvestre C, Clough K, Asselain B et al. Axillary treatment in conservative management of operable breast cancer: dissection or radiotherapy? Results of a randomized study with 15 years of follow-up. J Clin Oncol 2004; 22: 97-101.

9. Fisher $\mathrm{B}$, Jeong JH, Anderson S et al. Twenty-five-year follow-up of a randomized trial comparing radical mastectomy, total mastectomy and total mastectomy followed by irradiation. N Engl J Med 2002; 437: 567-575.

10. Donker M, van Tienhoven G, Straver ME et al. Radiotherapy or surgery of the axilla after a positive sentinel node in breast cancer (EORTC 10981-22023 AMAROS): a randomised, multicentre, open-label, phase 3 non-inferiority trial. Lancet Oncol 2014; 15: 1303-1310.

11. Sávolt Á, Péley G, Polgár $C$ et al. Eight-year follow up result of the OTOASOR trial: The optimal treatment of the axilla — surgery or radiotherapy after positive sentinel lymph node biopsy in early-stage breast cancer: A randomized, single centre, phase III non-inferiority trial. Eur J Surg Oncol 2017; 43: 672-679.

12. Poortmans PM, Collette $\mathrm{S}$, Kirkove $\mathrm{C}$ et al; EORTC Radiation Oncology and Breast Cancer Groups. Internal mammary and medial supraclavicular irradiation in breast cancer. N Engl Med. 2015; 373: 317-327.

13. Whelan TJ, Olivotto IA, Parulekar WR et al; MA.20 Study Investigators Regional nodal irradiation in early-stage breast cancer. N Engl J Med 2015; 373: 307-316.

14. Morrow M, van Zee KJ, Patil S et al. Axillary dissection and nodal irradiation can be avoided for most node-positive Z0011-eligible breast cancers: a prospective validation study of 793 patients. Ann Surg 2017; 266: 457-462.

15. Pilewskie $M$, Zabor EC, Mamtani A et al. The optimal treatment plan to avoid axillary lymph node dissection in early-stage breast cancer patients differs by surgical strategy and tumor subtype. Ann Surg Oncol 2017; 24: 3527-3533.

16. Zhang J, Wang C. Axillary radiotherapy: an alternative treatment option for adjuvant axillary management of breast cancer. Sci Rep 2016; 6: 26304. doi: $10.1038 /$ srep26304. 